\title{
Orthogonal Analysis Based Performance Optimization for Vertical Axis Wind Turbine
}

\author{
Lei Song, ${ }^{1,2}$ Hong-Zhao Liu, ${ }^{1}$ and Zong-Xiao Yang ${ }^{2}$ \\ ${ }^{1}$ Faculty of Mechanical and Precision Instrument Engineering, Xi'an University of Technology, Xi'an 710048, China \\ ${ }^{2}$ College of Mechatronics Engineering, Henan University of Science and Technology, Luoyang 471003, China
}

Correspondence should be addressed to Hong-Zhao Liu; liu-hongzhao@163.com

Received 6 November 2015; Revised 26 January 2016; Accepted 1 February 2016

Academic Editor: Sachin Nimbalkar

Copyright (C) 2016 Lei Song et al. This is an open access article distributed under the Creative Commons Attribution License, which permits unrestricted use, distribution, and reproduction in any medium, provided the original work is properly cited.

\begin{abstract}
Geometrical shape of a vertical axis wind turbine (VAWT) is composed of multiple structural parameters. Since there are interactions among the structural parameters, traditional research approaches, which usually focus on one parameter at a time, cannot obtain performance of the wind turbine accurately. In order to exploit overall effect of a novel VAWT, we firstly use a single parameter optimization method to obtain optimal values of the structural parameters, respectively, by Computational Fluid Dynamics (CFD) method; based on the results, we then use an orthogonal analysis method to investigate the influence of interactions of the structural parameters on performance of the wind turbine and to obtain optimization combination of the structural parameters considering the interactions. Results of analysis of variance indicate that interactions among the structural parameters have influence on performance of the wind turbine, and optimization results based on orthogonal analysis have higher wind energy utilization than that of traditional research approaches.
\end{abstract}

\section{Introduction}

Wind energy is a renewable energy without pollution and will be an important resource for future green energy. There are two primary categories of modern wind turbines classified as horizontal axis wind turbine (HAWT) and vertical axis wind turbine (VAWT). With rapid development of the technologies of the permanent magnet generators, drag type VAWTs equipped with these generators can generate electricity within low wind speed ranges continuously because they have advantages such as very low start-up wind speed and high torque levels. However, wind energy utilization of the drag type VAWs is usually lower than that of other wind turbines; scholars do lots of studies to increase system efficiency for them.

Research approaches for the drag type VAWTs mainly include two categories, one is to change the structure and another is to add attachments. Both of them aim to improve wind energy utilization of VAWTs. Considering costs of prototypes, performance of wind rotor has been researching at different direction to improve its capability using experimental and numerical study method [1-4]. Studies on Savonius wind rotor usually focus on performance of the wind rotor itself and some attachments. The geometry of the blade shape (skeleton line) of Savonius rotor with two blades was optimized in order to obtain better performance of output power and static torque [5]. Attachments such as deflector plate were also used on Savonius wind rotor to improve wind energy collection [6, 7]. Considering performance of threedimensional structure of Savonius wind rotor, height aspect ratio was considered and helical shape of blades was discussed and tested $[8,9]$. Considering costs of test equipment and prototypes, Computational Fluid Dynamics (CFD) methods are often rather used to analyze performance of wind rotor than wind tunnel tests. The applicability of Darrieus airfoil's design processes was discussed, especially for the approaches based on Panel and the CFD method [10]. Based on research results of scholars, we propose a novel type of VAWT wind rotor to improve the flow field and wind energy utilization, which is named as Fish-ridged vertical axis wind rotor.

When considering performance of kinds of wind rotors, characteristics such as shape of blades, number of blades, angle of installation, and obstacle shielding are usually studied, respectively, and then corresponding conclusions are 
drawn. However, structure of a wind rotor is constructed by multiple geometric shapes, that is, structural parameters; then, there is combined action of the structural parameters on performance of the wind rotor. Change of a structural parameter must influence other adjacent parameters; thus, there is interaction among them. Therefore, when considering improving performance of a wind rotor, the combined action and interaction of the parameters should not be ignored. Then, more than one factor should be considered simultaneously in design or analysis phases of a VAWT. Orthogonal experiment design is a solution for a problem with multiple factors and levels. Based on orthogonality, part of typical test points, which have characteristics of uniformity and dispersity, are selected to have a test in this method. It is an efficient, fast, and economic method of experimental design and can be used in various different fields. An approach combining fluid-structure interaction with an improved orthogonal experiment method was used to make an optimum design for a novel hybrid journal bearing with multiple factors of length, width, and depth of shallow recess and the width of deep recess [11]. An orthogonal experiment was carried out to investigate the influence of various parameters of the marine rotary desiccant A/C system [12]. An orthogonal experimental design method combined with quantitative analysis of small-angle X-ray scattering (SAXS) pattern was applied to optimize the synthesis of bioactive glasses. The number of trials for preparing MBGs could be greatly reduced and the primary factors affecting the formation of mesoporous structure and the properties of MBGs could be easily found out by orthogonal experimental design method [13]. Large Eddy Simulation (LES) field model was used as simulation model for fire analyses, and orthogonal experimental design (OED) method with a $L_{16}\left(2^{15}\right)$ orthogonal array was used as a tool to reduce the simulation runs in fire accident reconstruction [14]. An optimization study on the production of biodiesel from Camelina seed oil using alkaline transesterification was proposed, and the optimization was based on sixteen wellplanned orthogonal experiments $\left(O A_{16}\right.$ matrix) [15].

Considering the multiple structural parameters as factors, we use an orthogonal experiment method to exploit influence of interactions of structural parameters on wind energy utilization of the proposed wind rotor. The results are also compared with those of a single parameter optimization method to find out optimal combination of the parameters in this paper.

\section{Geometry and Simulation Model of the Rotor}

2.1. Structure of the Fish-Ridged Vertical Axis Wind Rotor. Savonius wind rotor is the most used drag type VAWTs because it has excellent performance of simple structure, fast start-up, and running at low wind speed; however, the power coefficient is lower than that of other types of rotors. To overcome the defects of traditional Savonius wind rotor, a novel VAWT is proposed by changing geometric shapes and number of blades, aiming at improving performance of wind resistance and torque. As shown in Figure 1, the blade

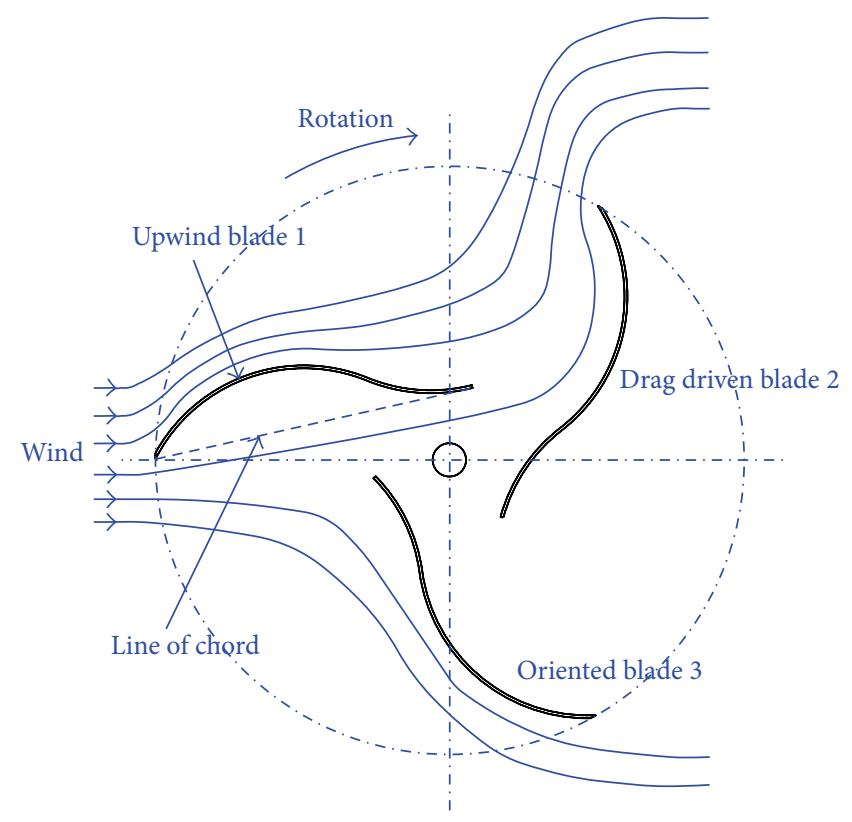

FIGURE 1: The supposed streamline of the Fish-ridged rotor.

is composed of two arcs which are connected smoothly with each other. In order to improve sharp bending streamlines occurring in overlap area of Savonius rotor with two blades, the novel rotor is composed of three blades in order to adjust angle of accepting airflow of the oriented blade. Similar to Savonius rotor, the inner space surrounded by three small arcs is defined as overlap area. Supposing airflow passes through blade 1, part of it is separated and guided by convex face of the blade and flows toward concave face of blade 2 to play a role as drag driven mode at this position. The other airflow is separated to the other side of blade 1 and part of it passes through the overlap area of the rotor to the concave face of blade 2 to drive it. Wind resistance of blade 3 decreases because the cut-in angle changes. Thus, the streamlines pattern is smoother than that of Savonius rotor when airflow passes through overlap area, which decreases blockage effects of counterrotating vertices and recirculating flow. When the rotor rotates around its axis, three blades act as guiding roles in turn at different time to change direction of wind. The function of small arcs of the three blades is to guide airflow to the next blade at an appropriate angle. This structure can not only decrease resistant torque formed by impact from airflow to convex face of the returning blade but also improve and enhance impact acting by airflow on concave faces by using airflow twice. Since the shape of each blade is similar to ridge of a swimming fish, it is named as Fish-ridged vertical axis wind rotor.

2.2. Parameters Selection. There are many structural parameters influencing performances of the wind rotor, such as blade shape and ratio of height to diameter. To obtain optimal structure of wind rotor with high wind energy conversion efficiency, tests by kinds of prototypes are unwise because of high costs. Computational Fluid Dynamics (CFD) simulation is an effective method to research performance and obtain 


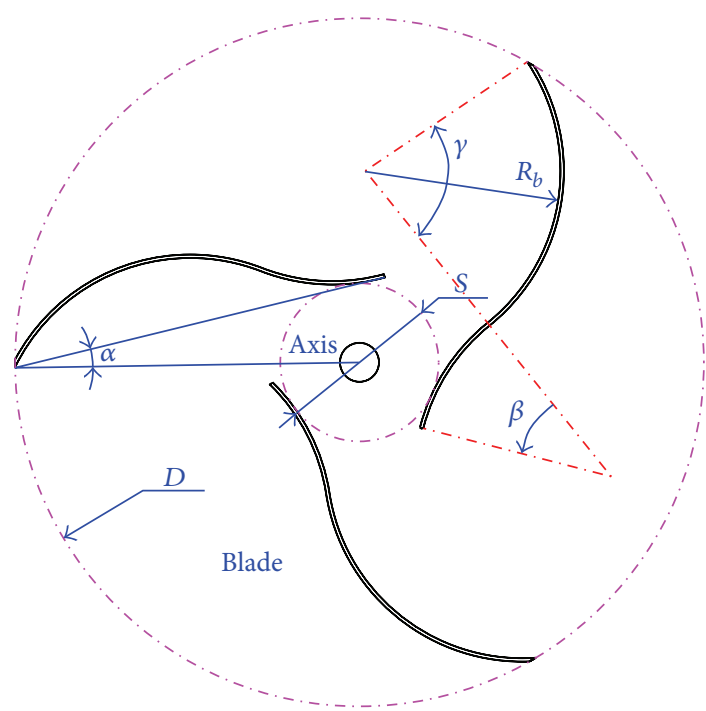

FIGURE 2: The main structure parameters for simulation.

optimal structure of the wind rotor. Since the cross-sectional shapes of the Fish-ridged wind rotor at different positions are the same, parameters which are located in a two-dimensional plane and flow field characteristics of the wind rotor are considered to be optimized. When diameter of the wind rotor $D$ is constant, the most important parameters influencing performance include radius of curvature $R_{b}$, installation angle $\alpha$, and central angle of small $\operatorname{arc} \beta$, as shown in Figure 2. In researches of lift-type VAWTs, there exist assumptions such as no interactions between two blades since they are far from each other; however, the assumptions are not suitable in researches of drag type VAWTs because the blades are designed closely. Thus, the parameters affect the wind energy utilization simultaneously and have effect on each other. A simple thinking is considering effect on the wind energy utilization of one structure parameter separately and then considering the interaction effects among the parameters on the wind energy utilization.

2.3. CFD Simulation Model of the Wind Rotors. At present, research approaches for the VAWTs mainly include wind tunnel tests and CFD methods. Considering costs of test equipment and prototypes, wind tunnel tests are usually used to test performance of a wind turbine and CFD methods are more suitable for analyzing performance and optimizing structural parameters of a wind turbine before wind tunnel tests.

Air can be seen as a two-dimensional incompressible flow. Thus, two-dimensional incompressible Navier-Stokes equations can be established as

$$
\begin{gathered}
\frac{\partial(\rho u)}{\partial t}+u \frac{\partial(\rho u)}{\partial t}+\nu \frac{\partial(\rho u)}{\partial y} \\
=-\frac{\partial p}{\partial x}+\mu\left(\frac{\partial^{2} u}{\partial x^{2}}+\frac{\partial^{2} u}{\partial y^{2}}\right)
\end{gathered}
$$

$$
\begin{gathered}
\frac{\partial(\rho v)}{\partial t}+u \frac{\partial(\rho v)}{\partial t}+v \frac{\partial(\rho v)}{\partial y} \\
=-\frac{\partial p}{\partial y}+\mu\left(\frac{\partial^{2} v}{\partial x^{2}}+\frac{\partial^{2} v}{\partial y^{2}}\right)
\end{gathered}
$$

$\rho$ is density of air, $t$ is lapse time, $u$ is velocity of the $X$ direction, $v$ is velocity of the $Y$ direction, $p$ is pressure of an infinitesimal body fluid, and $\mu$ is dynamic viscosity.

The two-dimensional continuity equation is

$$
\frac{\partial u}{\partial x}+\frac{\partial v}{\partial y}=0
$$

Simulation accuracy usually is the particular concern for performance analysis of VAWTs. Complex flow with turbulent and laminar features should be considered in modeling of the flow field. Standard $k-\varepsilon$ model is established for turbulent flow with fully developing turbulence and suitable for turbulent simulation model with high Reynolds number. Standard $k-\varepsilon$ model with standard wall condition was used as the turbulence closure model that solves two separate transport equations for a combined three-blade Darrieus Savonius wind rotor, since the model is fast and works well for flow with recirculation [16]. When Standard $k-\varepsilon$ model is used on flow with highly swirling flow, a certain degree of distortion will happen. RNG $k-\varepsilon$ model can deal with flow fields involving large flow separations better than Standard $k-\varepsilon$ model. Howell applied the RNG $k-\varepsilon$ turbulence model to a study of three-blade Darrieus wind turbine simulation, and the result agreed well with tunnel experiments [17]. Based on the unsteady Reynolds-averaged Navier-Stokes equation and RNG $k-\varepsilon$ turbulence model, flow field performance for a $500 \mathrm{~W}$ Darrieus-type VAWT was investigated [18]. Another improved scheme similar to RNG $k-\varepsilon$ model is Realizable $k-\varepsilon$ model, which can keep consistent with real turbulence for Reynolds stress because it satisfies the constraint condition of it. Detailed performance of a $2.5 \mathrm{~m}$ diameter VAWT was investigated by two-dimensional and complete three-dimensional simulations, using Realizable $k-\varepsilon$ model as turbulence modeling [19]. Essentially, RNG and Realizable $k-\varepsilon$ model are both for turbulent flow with fully developing turbulence with high Reynolds number; thus, wall functions should be considered to solve flow field near-wall region. Some scholars focus more interests on detailed inner flow field distribution and characteristics of a VAWT. $k-\omega$ turbulence model is more suitable for these problems since it includes influence of low Reynolds number, compressibility, and shear flow diffusion. Shear Stress Transport (SST) $k-\omega$ model combines advantages of calculation near-wall region by $k-\omega$ model and calculation in turbulent region by $k-\varepsilon$ model. The SST model has superior accuracy in separated or adverse pressure gradient flows simulations than using a Standard $k-\omega$ model or $k-\varepsilon$ model [20]. Performance of a classical and a Bach-type Savonius wind rotors is examined by means of quasi-2D flow predictions with SST $k-\omega$ model, and comparison revealed the importance of applying a laminarturbulent transition model [21]. Since main research purpose 
is generic effects of the wind rotor, the RNG $k$ - $\varepsilon$ two-equation turbulence model is adopted after algorithm effectiveness and economy of simulating time consumption has been investigated. The transport equations is

$$
\begin{aligned}
\frac{\partial(\rho k)}{\partial t}+\partial \frac{\left(\rho k u_{i}\right)}{\partial x_{i}} & =\frac{\partial}{\partial x_{i}}\left(a_{k} \mu_{\mathrm{eff}} \frac{\partial k}{\partial x_{i}}\right)+G_{k}+\rho \varepsilon \\
\frac{\partial(\rho \varepsilon)}{\partial t}+\partial \frac{\left(\rho \varepsilon u_{i}\right)}{\partial x_{i}}= & \frac{\partial}{\partial x_{i}}\left(a_{\varepsilon} \mu_{\mathrm{eff}} \frac{\partial k}{\partial x_{i}}\right)+\frac{C_{1 \varepsilon}^{*}}{k} G_{k} \\
& -C_{2 \varepsilon} \rho \frac{\varepsilon^{2}}{k} \\
\mu_{\mathrm{eff}}= & \mu+\mu_{t}, \\
\mu_{t}= & \rho C_{\mu} \frac{k^{2}}{\varepsilon}, \\
C_{1 \varepsilon}^{*} & =C_{1 \varepsilon}-\frac{\eta\left(1-\eta / \eta_{0}\right.}{1+\beta \eta^{3}} \\
\eta & =\left(2 E_{i j} \times E_{i j}\right)^{1 / 2} \frac{k}{\varepsilon} \\
E_{i j} & =\frac{1}{2}\left(\frac{\partial u_{i}}{\partial x_{j}}+\frac{\partial u_{j}}{\partial x_{i}}\right)
\end{aligned}
$$

where $G_{k}$ represents the generation of turbulence kinetic energy owing to the mean velocity gradients. Quantities $a_{k}$ and $a_{\varepsilon}$ are, respectively, the inverse effective Prandtl numbers for $k$ and $\varepsilon . \mu_{t}$ is viscosity coefficients and $\mu$ is the dynamic viscosity of atmospheric air. $E_{i j}$ is time-averaged strain rate of flow. The value of $C_{\mu}$ is constant as $0.0845, C_{1 \varepsilon}$ is 1.42 , and $C_{2 \varepsilon}$ is 1.68 .

In the near-wall region of the wind rotor blades, the enhanced wall treatment approach is adopted in order to maximize the reliability of the RNG $k-\varepsilon$ turbulence model. The first element height is estimated by imposing the maximum nondimensional wall distance $y^{+}$value in all the rotor near-wall cells to be approximately 1 or less. $y^{+}$is evaluated by (4), where $u^{*}$ is the reference velocity and $\rho$ is the density. The near-wall fine meshes in this work are designed so that wall $y^{+}$is in good agreement with the requirement of approximate 1 :

$$
y^{+}=\frac{\Delta y u^{*} \mu}{\rho} .
$$

Usually, for the VAWTs which have the same cross section shapes at different height direction, a two-dimensional simulation model was suitable because the flow field distributions are the same at most layers except for the bottom and top sides $[22,23]$. A flow visualization technique with smoke wires was also applied on Savonius type wind turbines to investigate flow field distribution [24]. When energy dissipation at the bottom and top sides of a VAWT should be considered, or cross section shapes were not the same at different height direction such as $\Phi$-shaped VAWT, a three-dimensional simulation model was applied $[25,26]$. When simulation model changes from two-dimensional to three-dimensional,

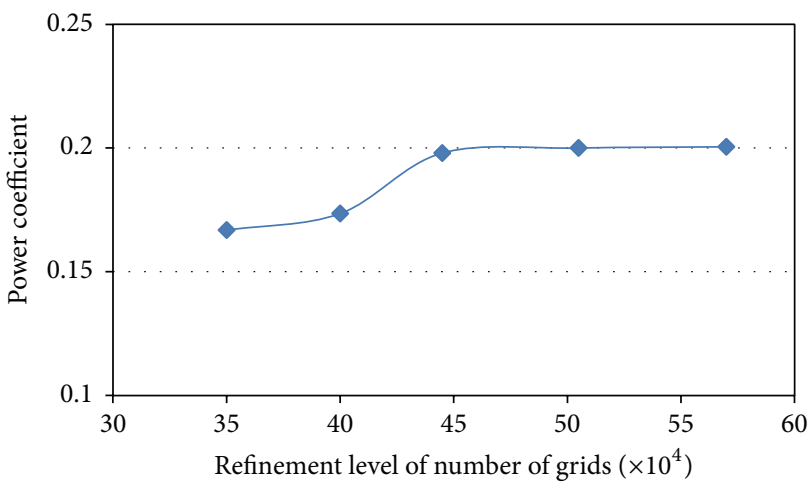

Figure 3: Power coefficient versus number of elements for GIL.

the total number of grids and simulation times increases tempestuously. Considering costs of simulation times, a twodimensional simulation model of the wind rotor is used in this paper.

For transient numerical simulation, independence between number of grids and simulation result should be determined, which is call Grid Independent Limit (GIL). The computations is firstly carried out with five levels of number of elements with about 50,000 intervals, and the power coefficient of the wind rotor is taken as the parameter for which GIL is determined. Most changes of number of elements occur in rotating region of the overall domain for a sliding mesh simulation method. Each level is solved in Fluent with the same set of input parameters with $12.4 \mathrm{~m} / \mathrm{s}$ of wind speed and $193.2 \mathrm{rpm}$ of rotating speed, whose data are from wind tunnel experiments. The grid independence analysis of the wind rotor with various levels of number of elements is shown in Figure 3. When number of elements is lower than level of 450,000, simulation result of power coefficient is susceptible to it. While number of elements exceeds this level, change of power coefficient value is very small. Thus, a level of about 480,000 elements is considered and set for final simulation. Meanwhile, the simulation is set to unsteady with a time step equal to the time needed for the rotor to perform an azimuthal rotation of $1^{\circ}$.

The computational domain is set rectangular and a long flow field area at the back of the wind rotor is set as shown in Figure 4(a) considering the aerodynamic performance of large wake flow effects in the wind rotor. Diameter of the wind rotor is set as $0.7 \mathrm{~m}$ which is consistent with size of the prototype and height is the same value. Considering the division of grids, boundary conditions, computer resources, and other factors, the inlet length of computational domain which locates at left side of the domain is 10 times as much as the rotor diameter; the distance from center of the wind rotor to outlet is 15 times and the width is 20 times. The computational domain is considered discretized as unstructured triangular elements. The mesh distribution near the rotors is as shown in Figure 4(b). A boundary layer mesh is used to achieve accurate results, as shown in Figure 4(c).

Sliding mesh (SM) is widely used in engineering for good simplicity and applicability. In the process of CFD, RNG 


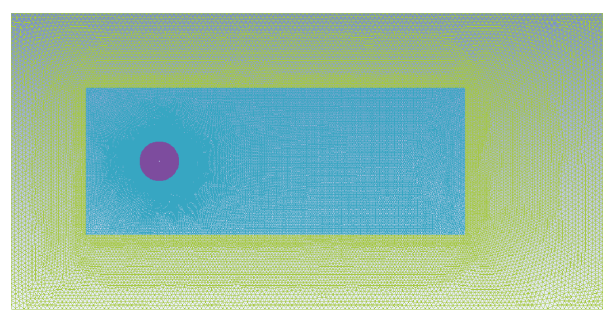

(a) Overall computational domain

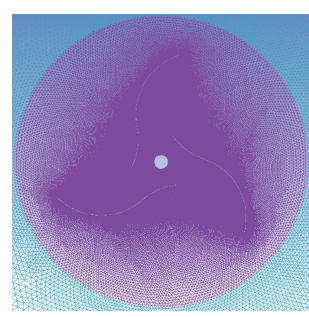

(b) Fish-ridged rotor

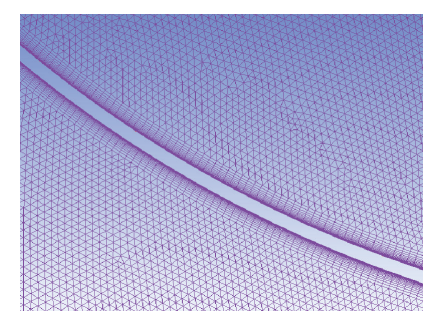

(c) Close-up of boundary mesh

FIgURE 4: Two-dimensional trimesh of the rotor.

$k-\varepsilon$ viscous model is used, and SIMPLE scheme of pressurevelocity coupling and second-order upwind in spatial discretization are selected to solve the simulation. The circle in Figure 4 is defined as interface, and blades are defined as moving wall with rotational motion. Inlet is velocityinlet type and outlet is outflow type with operating pressure value of 101325 Pascal. Considering performance of matched permanent magnet generator, revolving speed of the rotor is selected as $193.2 \mathrm{rpm}$ when wind speed is $12.4 \mathrm{~m} / \mathrm{s}$ with load of generator, whose data are from experiments.

\section{Results and Discussion}

3.1. Single Parameter Optimization Method for the Fish-Ridged Wind Rotor. Original dimensions of the wind rotor are that $R_{b}$ is $200 \mathrm{~mm}, \alpha$ is $13.1^{\circ}$, and angle $\beta$ is $36.5^{\circ}$ corresponding to a prototype model. Lengths of the two arcs in a blade are different, but the radii of them are set to be equal to each other in order to simplify the structure. Values of variables are set the as same interval. Near the range of optimal results, a method of interpolation is used to improve the accuracy. Power coefficient of the wind rotor $\eta$ is calculated as (5). $T_{r}$ is torque, $n_{r}$ is revolving speed $(\mathrm{rpm}), \rho$ is air density $\left(1.225 \mathrm{~kg} / \mathrm{m}^{3}\right), A_{r}$ is rotor swept area, and $v$ is wind speed:

$$
\eta=\frac{T_{r} \times n_{r}}{9550 \times 0.5 \times \rho \times A_{r} \times v^{3}} .
$$

Function of parameter radius of curvature $R_{b}$ is to create driving force of the wind rotor. In order to drive the wind rotor to rotate, drag force in advancing blade should be different from that in returning blade; that is, drag coefficients of the two blades are different, which is caused by radius of curvature $R_{b}$ of blade. Radius of curvature $R_{b}$ refers to the large arc segment in a blade which causes drag force mainly. Keeping the rest of the structure parameters constant, radius of curvature $R_{b}$ of the large arc is changed to study its influence on wind energy conversion efficiency, while connection between the large and small arc segments is kept smooth as far as possible. Methods of modeling and simulation are as mentioned above; the result is shown in Figure 5. Power coefficient of wind rotor obtains a peak value at about $23.1 \%$ in simulation while $R_{b}$ is $192.5 \mathrm{~mm}$. Shape of blades trends to be a semicircle and to increase power coefficient when $R_{b}$ decreases, but it has a baneful influence to construct overlap area $S$; thus, power coefficient decreases. Large arc segment

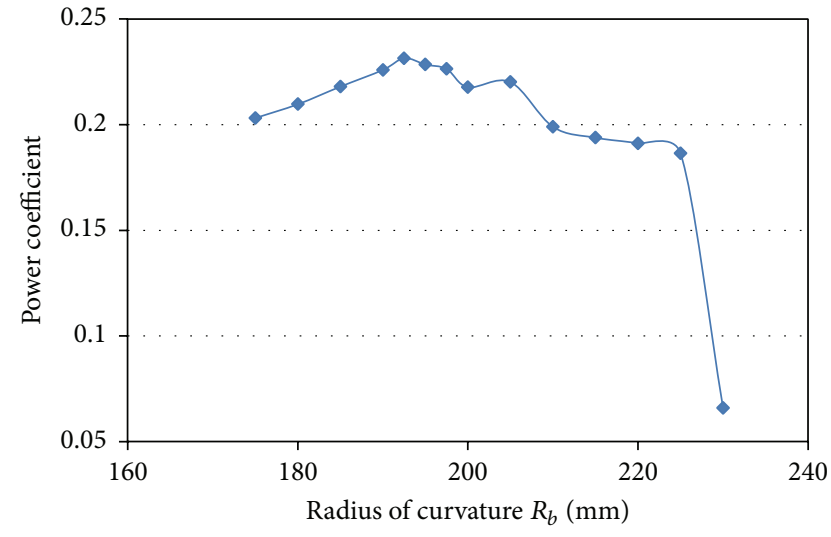

Figure 5: Simulation for radius of curvature.

will tend to become a plane when $R_{b}$ increases, and difference of drag coefficient between advancing and returning blades decreases; thus, power coefficient decreases also.

Influence of installation angle $\alpha$ on wind power conversion coefficient mainly aims to construct upwind angle and overlap area $S$ so as to keep airflows from windward to leeward with appropriate velocity and flux. $\alpha$ is defined as intersection angle between line connected by two endpoints of a blade and line connected by outer endpoint of the blade and center of the axis, as shown in Figure 2. Simulation result for installation angle is shown in Figure 6. Peak value of the wind rotor power coefficient occurs when $\alpha$ is $10.1^{\circ}$. Power coefficient decreases while $\alpha$ decreases from peak value, which is caused by reduction of overlap area $S$ which reduces and thus less wind flow passes through $S$ to leeward side to drive the second blade. While $\alpha$ increases, the wind rotor tends to become a lift-driven type rotor and influence of $S$ vanishes gradually; thus, power coefficient decreases also.

The purpose of central angle of small arc $\beta$ is to form the overlap area $S$ and its outlet shape. Convex side of the small arc can guide airflow out of overlap area with appropriate streamline; meanwhile, concave side of the small arc should not block airflow driving another blade. Simulation for $\beta$ versus wind rotor power coefficient is shown in Figure 7. Peak value of power coefficient occurs when $\beta$ is $30^{\circ}$. With $\beta$ decreasing, effective guidance function of overlap area $S$ reduces because $S$ cannot be formed and thus wind flow cannot be guided from windward side to leeside to drive another blade once more. While $\beta$ increases, the angle that 
TABLE 1: Simulation results for different structural size of wind rotors.

\begin{tabular}{|c|c|c|c|c|c|c|}
\hline Simulation & $R_{b}(\mathrm{~mm})$ & $\alpha\left(^{\circ}\right)$ & $\beta\left(^{\circ}\right)$ & Torque $(\mathrm{Nm})$ & Power $(\mathrm{W})$ & Power coefficient $(\%)$ \\
\hline Original parameters & 200 & 13.1 & 36.5 & 5.62 & 113.79 & 19.98 \\
\hline Three parameters' combination & 192.5 & 10.1 & 30 & 6.76 & 136.84 & 24.03 \\
\hline
\end{tabular}

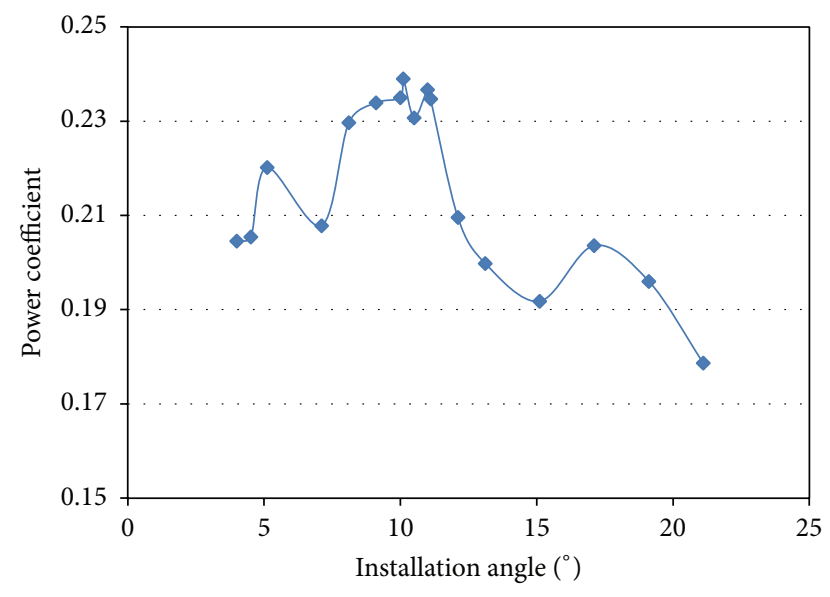

FIGURE 6: Simulation for installation angle.

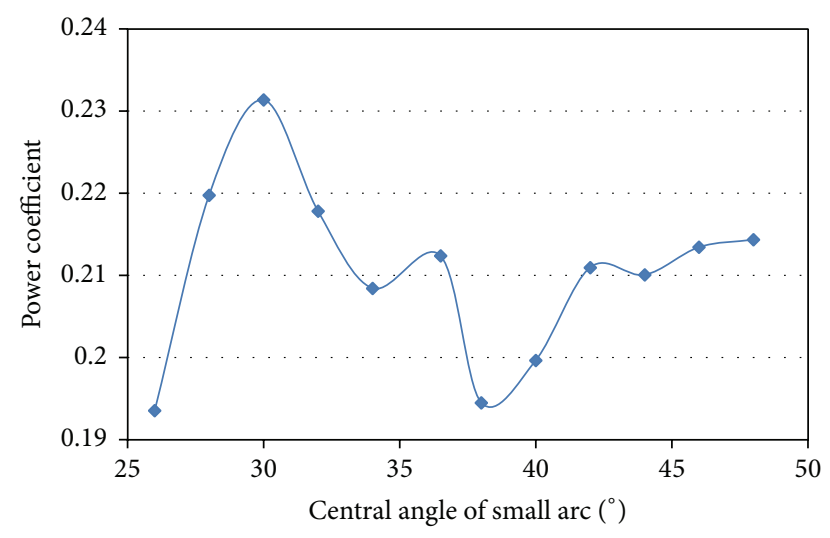

Figure 7: Simulation for central angle of small arc.

causes airflow toward the large arc segment of the second blade is not fit to form larger drag force. Hence, power coefficient tends to decrease in the two cases.

To obtain optimal power coefficient of the wind rotor, those three optimal parameters are combined to construct a new rotor model to simulate for its performance. Results are shown in Table 1. Power coefficient of new wind rotor with three optimal parameters is $24.03 \%$ and higher than that with original size at $19.98 \%$ in simulation. It shows that the new wind rotor with three optimal parameters has better performance than the former. However, the optimization result considers the effect of each structural parameter on wind energy utilization separately, and the interaction among them is not considered. For instance, the installation angle and the central angle of small arc construct the overlap area together; when a parameter value is changed, another parameter is inevitably under the influence. Thus, the optimization result partly reflects the influence of the change of structure parameters.

3.2. Orthogonal Analysis of the Structural Parameters. As discussed above, structural parameters of the Fish-ridged wind rotor have significant influence on the wind energy utilization. Meanwhile, there are interactions among the parameters and effect on the wind energy utilization combing the parameters together. In order to study the influence among the structural parameters and that on the wind energy utilization with more than one parameter, the parameters can be considered as influence factors on wind energy utilization. When there are many investigated factors and each factor has many values, orthogonal experimental design is suitable for exploring the influence among the factors. By selecting the right amount of representative points from a large number of test points, orthogonal experimental method is used to arrange the tests and to analyze the data using the stated orthogonal table. By integrated designing, comprehensive comparing, and statistical analyzing, the method can find out better design result. Because orthogonal table has characteristics of balance and dispersion by balanced sampling in the range of factors, each test in the table has strong representative; thus, the tests are able to achieve the purpose of the experiment. Based on the optimal results of structural parameters in single parameter optimization processes, orthogonal experiment method is used to analyze the influence of the parameters on wind energy utilization and to explore the best combination of them by variance components method.

The influential factors of the Fish-ridged wind rotor have been simplified as radius of curvature, installation angle, and chord length of small arc in the previous section. Then, they can be the three factors in orthogonal analysis. In works of single parameter optimization processes, each optimal result of the parameters has been obtained and the combination of them shows higher wind energy utilization in Table 1. Since there are interactions among the parameters, the combination parameters may not be the best optimal results. Thus, the distribution of levels for each factor can be arranged to consider the single parameter optimal result as the center and to spread to both sides of it. Considering the combination of parameters is a relative optimal result which is obtained by single parameter optimization results, three levels for each factor are determined in this study, whose values are based on consideration of the prototype. The factors and levels are shown in Table 2.

Interactions that influence the targets of the experiment significantly should be considered to be selected, which rely on the professional knowledge and experience. If the influence degree of the interactions cannot be determined, they also should be added to the orthogonal table and then 
TABLE 2: Factors and levels of orthogonal experiment.

\begin{tabular}{lcccc}
\hline & Factors & & \multicolumn{3}{c}{ Levels of factor } \\
& & 1 & 2 & 3 \\
\hline$A$ & $\alpha\left(^{\circ}\right)$ & 8.6 & 10.1 & 11.6 \\
$B$ & $R_{b}(\mathrm{~mm})$ & 187.5 & 192.5 & 197.5 \\
$C$ & $\beta\left(^{\circ}\right)$ & 27.5 & 30.0 & 32.5 \\
\hline
\end{tabular}

it should be determined whether the effect is remarkable by analyzing the test results. Through theoretical analysis, the selected intersections include intersection between $\alpha$ and $\beta$, intersection between $\alpha$ and $R_{b}$, and that between $R_{b}$ and $\beta$.

Selection of orthogonal table should consider number of factors and their levels. Usually, the sum of the number of factors and interactions should be less than the number of columns of orthogonal table; the number of levels of each factor should be equal to the number of levels corresponding to the orthogonal table, and interactions between factors with $n$ levels occupy $n-1$ columns. A smaller table will be selected when these conditions have been satisfied. For the problem with three factors and three levels for each factor, an orthogonal table of $L_{9}\left(3^{3}\right)$ has minimum test time. Considering accuracy of the test and total number of tests are not so much, an orthogonal table of $L_{27}\left(3^{13}\right)$ is determined in this study. There are 13 columns in the table and 27 times of test should be carried out. The factors which cannot to be ignored for interaction should be considered firstly when headers of the table are designed, including the factors that are not to be known whether to ignore or not. According to the principle of no mix, the factors and their interaction should be filled into header of the table. Then, the other factors whose interaction can be ignored can be randomly filled in the rest of the header. The orthogonal table with three factors and three levels for each factor of the test is shown as Table 3. Number means one test for a combination of levels of three factors in one row. $A, B$, and $C$ indicate the factors of $\alpha$, $R_{b}$, and $\beta$. The other nonnull columns indicate the interaction columns corresponding to the factors. The vacant columns without factors and interaction are used for error analysis. At least one vacant column is needed in the orthogonal table.

In order to estimate test errors, especially degree of importance that the factors influence the test results, analysis of variance (ANOVA) is used to analyze the interaction of structural parameters of the Fish-ridged wind rotor. The method is especially useful for the experiments that number of levels is equal to or greater than 3 and interaction should be considered. ANOVA aims to research which control variables have a significant influence on the observed variables by studying variance of the observed variables. Observed variables of the test results are simulation results of wind energy utilization by combining of levels of three factors, as shown in Table 3.

The total square sum of deviations $\mathrm{SS}_{T}$ is defined as (6). It reflects the total difference in the test results. The bigger the $\mathrm{SS}_{T}$ is, the greater the difference between test results is. One of the reasons is change of factors and levels. The variables of $P, Q$, and $T$ are shown as (7)-(9). $n$ is total test times in orthogonal analysis:

$$
\begin{aligned}
\mathrm{SS}_{T} & =\sum_{i=1}^{n} y_{i}^{2}-\frac{1}{n}\left(\sum_{i=1}^{n} y_{i}\right)^{2}=Q-P, \\
P & =\frac{1}{n}\left(\sum_{i=1}^{n} y_{i}\right)^{2}=\frac{T^{2}}{n}, \\
Q & =\sum_{i=1}^{n} y_{i}^{2} \\
T & =\sum_{i=1}^{n} y_{i} .
\end{aligned}
$$

The square sum of deviations $\mathrm{SS}_{j}$ caused by factors is calculated as (10). $r$ is number of levels. $K_{i}$ means the sum of test results at one column when number of the level is $i$. $k_{i}$ is arithmetic mean value of test results when the level of a factor at one column is $i$. It is calculated by $K_{i} / s . s$ is times that a level occurs at one column. $\bar{y}$ is arithmetic mean value of test results. The total square sum of deviations $\mathrm{SS}_{T}$ can be divided into that of different columns:

$$
\begin{aligned}
\mathrm{SS}_{j} & =\frac{n}{r} \sum_{i=1}^{r}\left(k_{i}-\bar{y}\right)^{2}=\frac{r}{n}\left(\sum_{i=1}^{r} K_{i}^{2}\right)-\frac{T^{2}}{n} \\
& =\frac{r}{n}\left(\sum_{i=1}^{r} K_{i}^{2}\right)-P .
\end{aligned}
$$

Square sum of deviations of test errors $\mathrm{SS}_{e}$ is total square sum of deviations corresponding to all the vacant columns and is calculated by

$$
\mathrm{SS}_{e}=\sum \mathrm{SS}_{\text {VacantColumns }} \text {. }
$$

Since interactions are considered as factors in orthogonal experiment design, they take up some columns. If only one column is taken up by an interaction, the square sum of deviations equals the $\mathrm{SS}_{j}$. If more than one column is taken up by one interaction, the square sum of deviations equals the sum of the square sum of deviations which is located at the taken-up columns. An instance is shown as

$$
\mathrm{SS}_{A \times B}=\mathrm{SS}_{(A \times B)_{1}}+\mathrm{SS}_{(A \times B)_{2}} .
$$

The total degree of freedom $\mathrm{df}_{T}$ is calculated by (13), and the degree of freedom corresponding to square sum of deviations of one column $\mathrm{df}_{j}$ is

$$
\begin{aligned}
\mathrm{df}_{T} & =n-1, \\
\mathrm{df}_{j} & =r-1 .
\end{aligned}
$$

The degree of freedom of interaction of two factors is the product of degree of freedom of two factors, as an instance in

$$
\mathrm{df}_{A \times B}=\mathrm{df}_{A} \times \mathrm{df}_{B} .
$$


TABLE 3: Orthogonal table and test results.

\begin{tabular}{|c|c|c|c|c|c|c|c|c|c|c|c|c|c|c|}
\hline \multirow{3}{*}{ No. } & \multicolumn{13}{|c|}{$\mathrm{F}$} & \multirow{3}{*}{ Test result (\%) } \\
\hline & 1 & 2 & 3 & 4 & 5 & 6 & 7 & 8 & 9 & 10 & 11 & 12 & 13 & \\
\hline & $A$ & $B$ & $(A \times B)_{1}$ & $(A \times B)_{2}$ & $C$ & $(A \times C)_{1}$ & $(A \times C)_{2}$ & $(B \times C)_{1}$ & $\mathrm{VC}_{1}$ & $\mathrm{VC}_{2}$ & $(B \times C)_{2}$ & $\mathrm{VC}_{3}$ & $\mathrm{VC}_{4}$ & \\
\hline 1 & 8.6 & 187.5 & (1) & (1) & 27.5 & (1) & (1) & (1) & (1) & (1) & (1) & (1) & (1) & 25.41 \\
\hline 2 & 8.6 & 187.5 & (1) & (1) & 30.0 & (2) & (2) & (2) & (2) & (2) & (2) & (2) & (2) & 21.95 \\
\hline 3 & 8.6 & 187.5 & (1) & (1) & 32.5 & (3) & (3) & (3) & (3) & (3) & (3) & (3) & (3) & 22.04 \\
\hline 4 & 8.6 & 192.5 & (2) & (2) & 27.5 & (1) & (1) & (2) & (2) & (2) & (3) & (3) & (3) & 21.14 \\
\hline 5 & 8.6 & 192.5 & (2) & (2) & 30.0 & (2) & (2) & (3) & (3) & (3) & (1) & (1) & (1) & 21.06 \\
\hline 6 & 8.6 & 192.5 & $(2)$ & (2) & 32.5 & (3) & (3) & (1) & (1) & (1) & (2) & (2) & (2) & 25.08 \\
\hline 7 & 8.6 & 197.5 & (3) & (3) & 27.5 & (1) & (1) & (3) & (3) & (3) & (2) & (2) & (2) & 20.17 \\
\hline 8 & 8.6 & 197.5 & (3) & (3) & 30.0 & (2) & (2) & (1) & (1) & (1) & (3) & (3) & (3) & 21.12 \\
\hline 9 & 8.6 & 197.5 & (3) & (3) & 32.5 & (3) & (3) & (2) & (2) & (2) & (1) & (1) & (1) & 21.24 \\
\hline 10 & 10.1 & 187.5 & (2) & (3) & 27.5 & (2) & (3) & (1) & (2) & (3) & (1) & (2) & (3) & 21.51 \\
\hline 11 & 10.1 & 187.5 & (2) & (3) & 30.0 & (3) & (1) & (2) & (3) & (1) & (2) & (3) & (1) & 20.53 \\
\hline 12 & 10.1 & 187.5 & (2) & (3) & 32.5 & (1) & (2) & (3) & (1) & (2) & (3) & (1) & (2) & 21.60 \\
\hline 13 & 10.1 & 192.5 & (3) & (1) & 27.5 & (2) & (3) & (2) & (3) & (1) & (3) & (1) & (2) & 27.40 \\
\hline 14 & 10.1 & 192.5 & (3) & (1) & 30.0 & (3) & (1) & (3) & (1) & (2) & (1) & (2) & (3) & 22.58 \\
\hline 15 & 10.1 & 192.5 & (3) & (1) & 32.5 & (1) & (2) & (1) & (2) & (3) & (2) & (3) & (1) & 23.03 \\
\hline 16 & 10.1 & 197.5 & (1) & (2) & 27.5 & (2) & (3) & (3) & (1) & (2) & (2) & (3) & (1) & 24.99 \\
\hline 17 & 10.1 & 197.5 & (1) & (2) & 30.0 & (3) & (1) & (1) & (2) & (3) & (3) & (1) & (2) & 22.31 \\
\hline 18 & 10.1 & 197.5 & (1) & (2) & 32.5 & (1) & (2) & (2) & (3) & (1) & (1) & (2) & (3) & 22.49 \\
\hline 19 & 11.6 & 187.5 & (3) & (2) & 27.5 & (3) & (2) & (1) & (3) & (2) & (1) & (3) & (2) & 21.87 \\
\hline 20 & 11.6 & 187.5 & (3) & (2) & 30.0 & (1) & (3) & (2) & (1) & (3) & (2) & (1) & (3) & 24.81 \\
\hline 21 & 11.6 & 187.5 & (3) & (2) & 32.5 & (2) & (1) & (3) & (2) & (1) & (3) & (2) & (1) & 22.58 \\
\hline 22 & 11.6 & 192.5 & (1) & (3) & 27.5 & (3) & (2) & (2) & (1) & (3) & (3) & (2) & (1) & 23.47 \\
\hline 23 & 11.6 & 192.5 & (1) & (3) & 30.0 & (1) & (3) & (3) & (2) & (1) & (1) & (3) & (2) & 22.40 \\
\hline 24 & 11.6 & 192.5 & (1) & (3) & 32.5 & (2) & (1) & (1) & (3) & (2) & (2) & (1) & (3) & 22.13 \\
\hline 25 & 11.6 & 197.5 & $(2)$ & (1) & 27.5 & (3) & (2) & (3) & (2) & (1) & (2) & (1) & (3) & 22.76 \\
\hline 26 & 11.6 & 197.5 & (2) & (1) & 30.0 & (1) & (3) & (1) & (3) & (2) & (3) & (2) & (1) & 21.51 \\
\hline 27 & 11.6 & 197.5 & $(2)$ & $(1)$ & 32.5 & $(2)$ & $(1)$ & $(2)$ & $(1)$ & (3) & $(1)$ & (3) & $(2)$ & 22.58 \\
\hline$K_{1}$ & 199.21 & 202.30 & 207.19 & 209.26 & 208.73 & 202.56 & 199.43 & 203.97 & 211.64 & 209.77 & 201.14 & 208.72 & 203.82 & $T=609.76$ \\
\hline$K_{2}$ & 206.44 & 208.29 & 197.77 & 206.33 & 198.27 & 205.32 & 199.35 & 205.61 & 198.92 & 199.01 & 205.45 & 201.34 & 205.36 & $Q=13843.02$ \\
\hline$K_{3}$ & 204.11 & 199.17 & 204.80 & 194.17 & 202.77 & 201.88 & 210.98 & 200.19 & 199.20 & 200.98 & 203.17 & 199.70 & 200.58 & $P=13770.64$ \\
\hline$k_{1}$ & 22.13 & 22.48 & 23.02 & 23.25 & 23.19 & 22.51 & 22.16 & 22.663 & 23.52 & 23.31 & 22.35 & 23.19 & 22.65 & \\
\hline$k_{2}$ & 22.94 & 23.14 & 21.97 & 22.93 & 22.03 & 22.81 & 22.15 & 22.85 & 22.10 & 22.11 & 22.83 & 22.37 & 22.82 & \\
\hline$k_{3}$ & 22.68 & 22.13 & 22.76 & 21.57 & 22.53 & 22.43 & 23.44 & 22.24 & 22.13 & 22.33 & 22.57 & 22.19 & 22.29 & \\
\hline$R$ & 0.804 & 1.013 & 1.047 & 1.677 & 1.161 & 0.382 & 1.292 & 0.604 & 1.414 & 1.196 & 0.479 & 1.002 & 0.531 & \\
\hline $\mathrm{SS}_{j}$ & 2.99 & 4.72 & 5.29 & 14.19 & 6.06 & 0.69 & 9.90 & 1.68 & 11.69 & 7.25 & 0.99 & 5.08 & 1.68 & \\
\hline
\end{tabular}

Then, the degree of freedom of error $\mathrm{df}_{e}$ is calculated by

$$
\mathrm{df}_{e}=\sum \mathrm{df}_{\text {VacantColumns }} \text {. }
$$

The mean square of a factor $\mathrm{MS}_{j}$ is calculated by (17). Values of $\mathrm{MS}_{j}$ of factors and interactions calculated from Table 3 are shown in Table 4:

$$
\mathrm{MS}_{j}=\frac{\mathrm{SS}_{j}}{\mathrm{df}_{j}}
$$

Since there exist relations that $\mathrm{MS}_{A \times C}$ is less than $\mathrm{MS}_{e}$ and $\mathrm{MS}_{B \times C}$ is less than $\mathrm{MS}_{e}$, this shows that interactions of $A \times C$ and $B \times C$ have less effect on the test results;
TABLE 4: Values of mean square.

\begin{tabular}{lcccccc}
\hline $\mathrm{MS}_{A}$ & $\mathrm{MS}_{B}$ & $\mathrm{MS}_{A \times B}$ & $\mathrm{MS}_{C}$ & $\mathrm{MS}_{A \times C}$ & $\mathrm{MS}_{B \times C}$ & $\mathrm{MS}_{e}$ \\
\hline 1.50 & 2.36 & 4.87 & 3.03 & 2.65 & 0.67 & 3.27 \\
\hline
\end{tabular}

thus, these interactions can be included into errors. Physical significance of $A \times C$ means interaction of $\alpha$ and $\beta$. Since $\alpha$ is used to construct overlap area $S$, it mainly influences diameter of $S$ and corresponding flow field characteristics. $\beta$ controls streamlines at outlet of $S$ and congestion degree when airflow passes through the small arc and exerts force on another blade. Thus, it is obvious that there is poor interaction between the two structural parameters, and the same 
TABLE 5: Contrast table of significance.

\begin{tabular}{lcccccc}
\hline Source of difference & $\mathrm{SS}_{j}$ & $\mathrm{df}_{j}$ & $\mathrm{MS}_{j}$ & $F_{j}$ & $F_{0.05}$ & Significance (relative) \\
\hline$A$ & 2.99 & 2 & 1.50 & 0.61 & 3.63 & \\
$B$ & 4.72 & 2 & 2.36 & 0.97 & 3.63 & III \\
$A \times B$ & 19.48 & 4 & 4.87 & 2.00 & 3.01 & $\mathrm{I}$ \\
$C$ & 6.06 & 2 & 3.03 & 1.24 & 3.63 & $\mathrm{II}$ \\
$e^{\Delta}($ Error $e, B \times C, A \times C)$ & 38.96 & 16 & 2.435 & & & \\
Total & 72.38 & 26 & & & & \\
\hline
\end{tabular}

situation is $B \times C$. New square sum of deviations of test errors, degree of freedom of error, and mean square of error should be recalculated by

$$
\begin{aligned}
\mathrm{SS}_{e}^{\Delta} & =\mathrm{SS}_{e}+\mathrm{SS}_{A \times C}+\mathrm{SS}_{B \times C}, \\
\mathrm{df}_{e}^{\Delta} & =\mathrm{df}_{e}+\mathrm{df}_{A \times C}+\mathrm{df}_{B \times C}, \\
\mathrm{MS}_{e}^{\Delta} & =\frac{\mathrm{SS}_{e}^{\Delta}}{\mathrm{df}_{e}^{\Delta}} .
\end{aligned}
$$

Then, $F$ value of factors and interactions can be calculated by (19) and the results are shown in Table 5. Usually, the greater the difference between $F$ value and the corresponding critical value is, the more significant the influence of the factor or interaction acting on the test results is, in other words, the more important the factor or interaction is. When level of significance $a$ is selected as 0.05 , the critical value $F_{\alpha}$ corresponding to factors and interactions can be looked up in $F$ distribution table and is shown in Table 5:

$$
F_{j}=\frac{\mathrm{MS}_{j}}{\mathrm{MS}_{e}} .
$$

Since $F_{j}$ is less than $F_{\alpha}$, it indicates that factors and interactions listed in Table 5 have no significant influence on test results. The main reason is that the test parameters in Table 2 are obtained from the single parameter optimization and values of test results have no drastic changes. From the viewpoint of effect on performance of wind rotor, the factor $A \times B$ has the relatively most important influence on test results. Physical significance of $A \times B$ means that when concave side of a blade faces directly the airflow, the other blade, whose convex side also faces the airflow, is at the location with more lower resistance coefficient compared with traditional Savonius wind rotor, because it has an angle with direction of the airflow. Meanwhile, the blade with concave face directly facing the airflow is working with top performance. It indicates that interaction of multiple factors or structural parameters must be considered when designing structure of a VAWT, which has often been neglected in the past. The factor $C$ also has important influence on test results relatively; it indicates that the added structure of small arc, compared with traditional Savonius wind rotor, also has important influence on wind energy utilization and can improve the performance of the wind rotor. Function of three small arcs is mainly to guide airflow out of overlap area smoothly and overcome phenomena of severe changing
TABle 6: Match table of $A \times B$ for wind energy utilization.

\begin{tabular}{lccc}
\hline Factor $B$ & & Factor $A$ & \\
& $8.6^{\circ}$ & $10.1^{\circ}$ & $11.6^{\circ}$ \\
\hline $187.5 \mathrm{~mm}$ & 23.13 & 21.21 & 23.09 \\
$192.5 \mathrm{~mm}$ & 22.43 & 24.34 & 22.67 \\
$197.5 \mathrm{~mm}$ & 20.84 & 23.26 & 22.28 \\
\hline
\end{tabular}

streamlines which often occurs in Savonius wind rotor. Because factor $B$ is mainly used to generate resistance to drive the wind rotor, sequence of the importance is $A \times B>C>B$ which shows that configuration and distribution of multiple blades have significant influence on design of VAWTs besides performance of blade.

$R$ in Table 3 is a range and can be calculated by (20) or (21) in a column. Value of $R$ indicates influence degree of a factor on the test results. A column with maximum value of $R$ shows that the levels of the factor have the biggest influence on the test results; that is, it is the most important factor. There is a relation of $R$ in Table 3 that $R_{c}>R_{b}>R_{a}$; thus, the sequence of factors according to their importance is $C$ of central angle of small arc, $B$ of radius of curvature, and $A$ of installation angle. Compared with traditional Savonius wind rotor, it also indicates that the added parts of small arcs on blades of the Fish-ridged wind rotor have significant influence on wind energy utilization:

$$
\begin{aligned}
& R=\max \left\{K_{1}, K_{2}, K_{3}\right\}-\min \left\{K_{1}, K_{2}, K_{3}\right\}, \\
& R=\max \left\{k_{1}, k_{2}, k_{3}\right\}-\min \left\{k_{1}, k_{2}, k_{3}\right\} .
\end{aligned}
$$

When considering function of interaction among the factors, the interactions $A \times B$ and $C$ have relatively important influence on wind energy utilization in Table 5. As shown in Table $3, K_{2}$ of factor $A$ comes to maximum value and $K_{2}$ of factor $B$ also, respectively. In match table of interaction of $A \times$ $B$ in Table 6, each table element is average value of test results for combination of the two factors in Table 3 and combination of $A_{2} B_{2}$ achieves the maximum value. Since $K_{1}$ of factor $C$ comes to maximum value in Table 3 , the optimal result can be determined as $A_{2} B_{2} C_{1}$; that is, radius of curvature $R$ is $192.5 \mathrm{~mm}$, installation angle $\alpha$ is $10.1^{\circ}$, and central angle of small arc $\beta$ is $27.5^{\circ}$. Since all of the combinations are designed in the orthogonal experiment, wind energy utilization of the combination $A_{2} B_{2} C_{1}$ can be observed to be $27.4 \%$ in Table 3 . The analysis results validate that interactions of structural parameters have indeed an influence on wind 
energy utilization of a wind rotor, and the added small arcs on the blades can improve performance of the Fish-ridged wind rotor compared with traditional Savonius wind rotor.

\section{Conclusions}

Similar to situation of Savonius wind rotor that has structural parameters of radius of curvature and diameter of central axis, the proposed Fish-ridged wind rotor, which aims to improve wind energy utilization compared with traditional drag type VAWTs, also has multiple structural parameters. In order to obtain optimal structure for smoothing flow field and improving wind energy utilization of the new wind rotor, there are interaction and combined action of the structural parameters. In this paper, a single parameter optimization approach is used to obtain optimal parameters to improve performance of the Fish-ridged wind rotor. Based on the single parameter optimization approach, an orthogonal experiment method is used to analyze the influence of interactions of the parameter factors on the wind energy utilization. The main conclusions include the following:

(1) In order to smooth flow field in the vicinity of the blades, multiple structural parameters should be considered and designed together; thus, influence on wind energy conversation by interaction of them will exist. By methods of orthogonal experiment design and variance analysis, the influence of interaction has been validated. For instance, in this paper, radius of curvature of blades and their installation angle are combined to construct optimal windward section and cut-in angle of airflow. Significance analysis shows the influence of the two structural parameters has important composite effect on purpose of improving performance of the wind rotor. The results also show that configuration and distribution of multiple blades have significant influence on design of VAWTs besides performance of blade. It provides reference for similar studies. For multiparameter optimization problems, if there are interactions or uncertainty interactions among parameters, they may be considered to obtain more accurate design results.

(2) Compared with blade of traditional Savonius wind rotor, there is an added structure of small arc which is connected with the big arc smoothly near the central axis. Square sum of deviations of central angle of the small arc is greater than other factors in orthogonal analysis showing that it also has important influence on wind energy utilization. The structure of three small arcs of the Fish-ridged wind rotor is to construct overlap area, which can smooth flow field in overlap area. The significance analysis also shows its influence on wind energy conversation and the best combination of the three factors obtains the highest wind energy utilization.

(3) The orthogonal analysis is based on the single parameter optimization results. By preliminary analysis for the test data, the test result of combination by orthogonal analysis is slightly bigger than that by single parameter optimization. When meticulous studies on performance of a wind rotor's structure is needed, combined action of structural parameters and their interaction should be considered. If the required accuracy in not high, respective optimization on each structural parameter can also be considered and used.

Studies in this paper are preliminary and future researches on such works may focus on those aspects:

(1) In the single parameter optimization processes, data of each structural parameter is distributed averagely in the interval. This may cause the situation that the optimal result may not locate at the real optimal point. Since the single parameter optimization method can also be used in structure design for wind rotor, solution can be modified where the golden section method is used to optimize a single structural parameter. This method can reduce calculation times and make the optimal result more closer to the real optimal point.

(2) In order to study influence of structural parameters on wind energy utilization, table model of orthogonal experiment is selected as $L_{27}\left(3^{13}\right)$ and is not a table with minimum amount of data. Considering the universality, the next work is to study influence of parameters on wind energy utilization based on small table so as to quickly obtain optimal structure without many more calculation times.

(3) Structure optimization based on single parameter optimization approach has been firstly used on a prototype of the Fish-ridged wind rotor with original structure parameters in order to obtain optimal structure collocation. A prototype of the wind rotor based on single parameter optimization results has also been manufactured and put to tests in wind tunnel environment in order to validate the performance. However, the interactions among the structural parameters have not been considered in the works, which are not in agreement with real situations of structure design. Thus, studies in this paper mainly pay attention to influence of interactions among the structural parameters on performance of the wind rotor. Since it is a systematic plan of performance analysis before and after structure optimizations and comparison between the proposed wind rotor and other traditional Savonius type wind rotors, at the next step, a prototype based on orthogonal analysis results will be manufactured to validate the studies stated in this paper.

\section{Conflict of Interests}

The authors declare that there is no conflict of interests regarding the publication of this paper. 


\section{Acknowledgment}

The financial support from the National Natural Science Foundation of China (NSFC) under Grants no. 71071078 and no. 51375145 is acknowledged gratefully.

\section{References}

[1] M. M. A. Bhutta, N. Hayat, A. U. Farooq, Z. Ali, S. R. Jamil, and Z. Hussain, "Vertical axis wind turbine-a review of various configurations and design techniques," Renewable \& Sustainable Energy Reviews, vol. 16, no. 4, pp. 1926-1939, 2012.

[2] V. D’Alessandro, S. Montelpare, R. Ricci, and A. Secchiaroli, "Unsteady Aerodynamics of a Savonius wind rotor: a new computational approach for the simulation of energy performance," Energy, vol. 35, no. 8, pp. 3349-3363, 2010.

[3] K. McLaren, S. Tullis, and S. Ziada, "Computational fluid dynamics simulation of the aerodynamics of a high solidity, small-scale vertical axis wind turbine," Wind Energy, vol. 15, no. 3, pp. 349-361, 2012.

[4] I. Ross and A. Altman, "Wind tunnel blockage corrections: review and application to Savonius vertical-axis wind turbines," Journal of Wind Engineering and Industrial Aerodynamics, vol. 99, no. 5, pp. 523-538, 2011.

[5] M. H. Mohamed, G. Janiga, E. Pap, and D. Thévenin, “Optimal blade shape of a modified Savonius turbine using an obstacle shielding the returning blade," Energy Conversion and Management, vol. 52, no. 1, pp. 236-242, 2011.

[6] B. D. Altan and M. Atılgan, "An experimental and numerical study on the improvement of the performance of Savonius wind rotor," Energy Conversion and Management, vol. 49, no. 12, pp. 3425-3432, 2008.

[7] K. Golecha, T. I. Eldho, and S. V. Prabhu, "Influence of the deflector plate on the performance of modified Savonius water turbine," Applied Energy, vol. 88, no. 9, pp. 3207-3217, 2011.

[8] M. A. Kamoji, S. B. Kedare, and S. V. Prabhu, "Performance tests on helical Savonius rotors," Renewable Energy, vol. 34, no. 3, pp. 521-529, 2009.

[9] A. Damak, Z. Driss, and M. S. Abid, "Experimental investigation of helical Savonius rotor with a twist of $180^{\circ}$," Renewable Energy, vol. 52, pp. 136-142, 2013.

[10] J. Chen, H. X. Yang, M. Yang, H. T. Xu, and Z. H. Hu, “A comprehensive review of the theoretical approaches for the airfoil design of lift-type vertical axis wind turbine," Renewable and Sustainable Energy Reviews, vol. 51, pp. 1709-1720, 2015.

[11] Q. Y. Lin, Z. Y. Wei, and N. Wang, "Optimum design of recess parameters for a high-speed hybrid journal bearing using fluidstructure interaction and improved orthogonal experiment method," Journal of the Balkan Tribological Association, vol. 21, no. 2, pp. 300-313, 2015.

[12] J. Zhu and W. Chen, "Energy and exergy performance analysis of a marine rotary desiccant air-conditioning system based on orthogonal experiment," Energy, vol. 77, pp. 953-962, 2014.

[13] L. Ji, Y. Si, H. Liu, X. Song, W. Zhu, and A. Zhu, "Application of orthogonal experimental design in synthesis of mesoporous bioactive glass," Microporous and Mesoporous Materials, vol. 184, pp. 122-126, 2014.

[14] P. Z. Yang, X. Tan, H. C. Sun, D. H. Chen, and C. Li, "Fire accident reconstruction based on les field model by using orthogonal experimental design method," Advances in Engineering Software, vol. 42, no. 11, pp. 954-962, 2011.
[15] X. Wu and D. Y. C. Leung, "Optimization of biodiesel production from camelina oil using orthogonal experiment," Applied Energy, vol. 88, no. 11, pp. 3615-3624, 2011.

[16] A. Ghosh, A. Biswas, K. K. Sharma, and R. Gupta, "Computational analysis of flow physics of a combined three bladed Darrieus Savonius wind rotor," Journal of the Energy Institute, vol. 88, no. 4, pp. 425-437, 2015.

[17] R. Howell, N. Qin, J. Edwards, and N. Durrani, "Wind tunnel and numerical study of a small vertical axis wind turbine," Renewable Energy, vol. 35, no. 2, pp. 412-422, 2010.

[18] Y. T. Lee and H. C. Lim, "Numerical study of the aerodynamic performance of a $500 \mathrm{~W}$ Darrieus-type vertical-axis wind turbine," Renewable Energy, vol. 83, pp. 407-415, 2015.

[19] M. S. Siddiqui, N. Durrani, and I. Akhtar, "Quantification of the effects of geometric approximations on the performance of a vertical axis wind turbine," Renewable Energy, vol. 74, pp. 661670, 2015.

[20] A. J. Buchner, M. W. Lohry, L. Martinelli, J. Soria, and A. J. Smits, "Dynamic stall in vertical axis wind turbines: comparing experiments and computations," Journal of Wind Engineering and Industrial Aerodynamics, vol. 146, pp. 163-171, 2015.

[21] K. Kacprzak, G. Liskiewicz, and K. Sobczak, "Numerical investigation of conventional and modified Savonius wind turbines," Renewable Energy, vol. 60, pp. 578-585, 2013.

[22] M. Kear, B. Evans, R. Ellis, and S. Rolland, "Computational aerodynamic optimisation of vertical axis wind turbine blades," Applied Mathematical Modelling, vol. 40, no. 2, pp. 1038-1051, 2016.

[23] G. Bedon, S. De Betta, and E. Benini, "A computational assessment of the aerodynamic performance of a tilted Darrieus wind turbine," Journal of Wind Engineering and Industrial Aerodynamics, vol. 145, pp. 263-269, 2015.

[24] M. Shaheen, M. El-Sayed, and S. Abdallah, "Numerical study of two-bucket Savonius wind turbine cluster," Journal of Wind Engineering and Industrial Aerodynamics, vol. 137, pp. 78-89, 2015.

[25] H. F. Lam and H. Y. Peng, "Study of wake characteristics of a vertical axis wind turbine by two- and three-dimensional computational fluid dynamics simulations," Renewable Energy, vol. 90, pp. 386-398, 2016.

[26] S. Joo, H. Choi, and J. Lee, "Aerodynamic characteristics of twobladed H-Darrieus at various solidities and rotating speeds," Energy, vol. 90, part 1, pp. 439-451, 2015. 


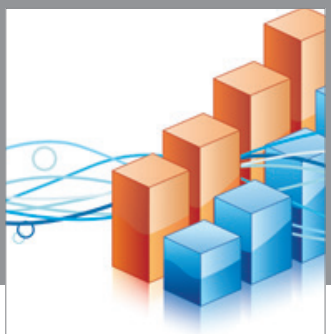

Advances in

Operations Research

vatem alat4

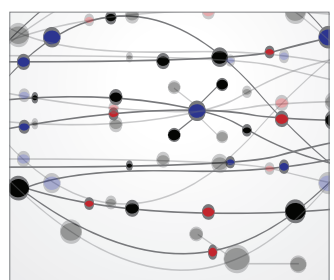

\section{The Scientific} World Journal
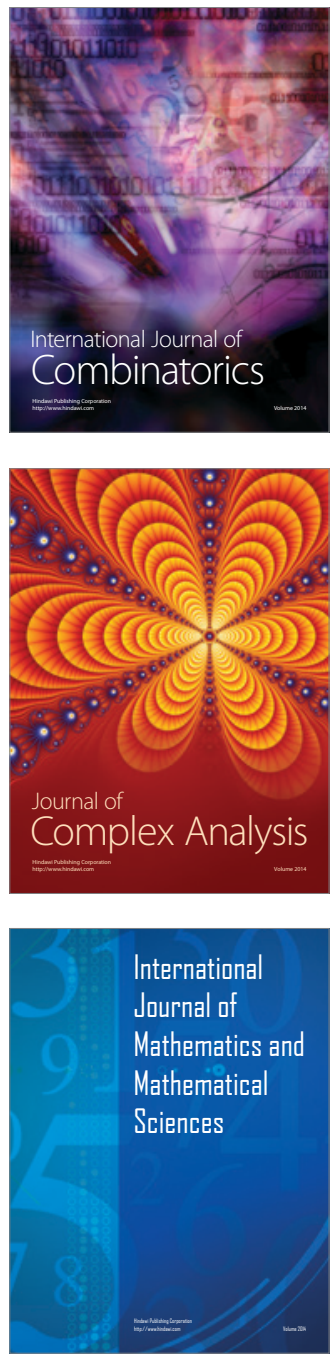
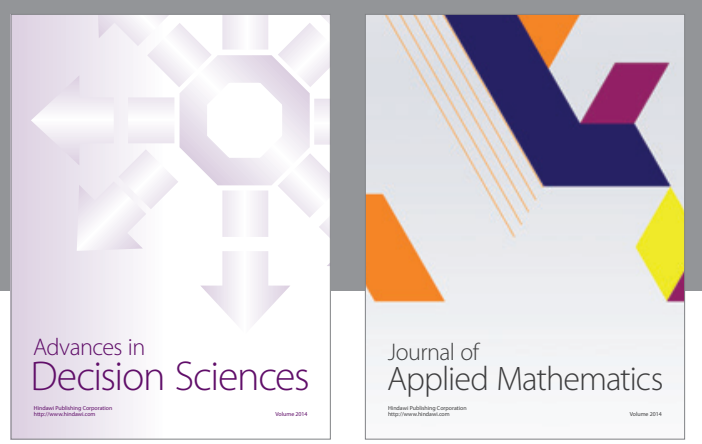

Algebra

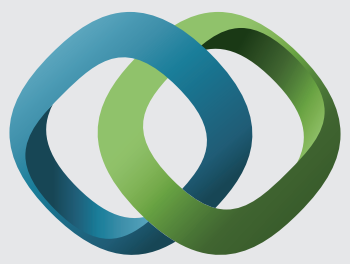

\section{Hindawi}

Submit your manuscripts at

http://www.hindawi.com
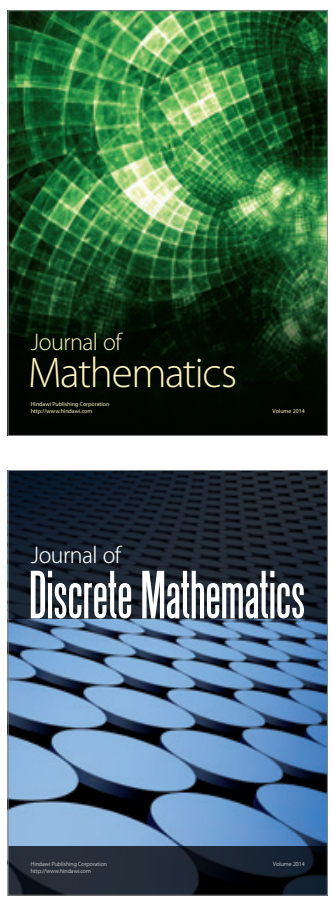

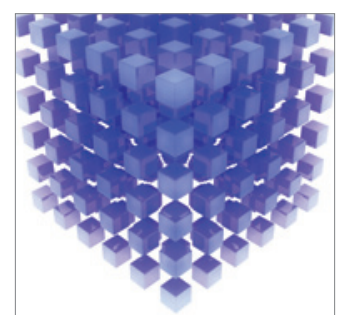

Mathematical Problems in Engineering
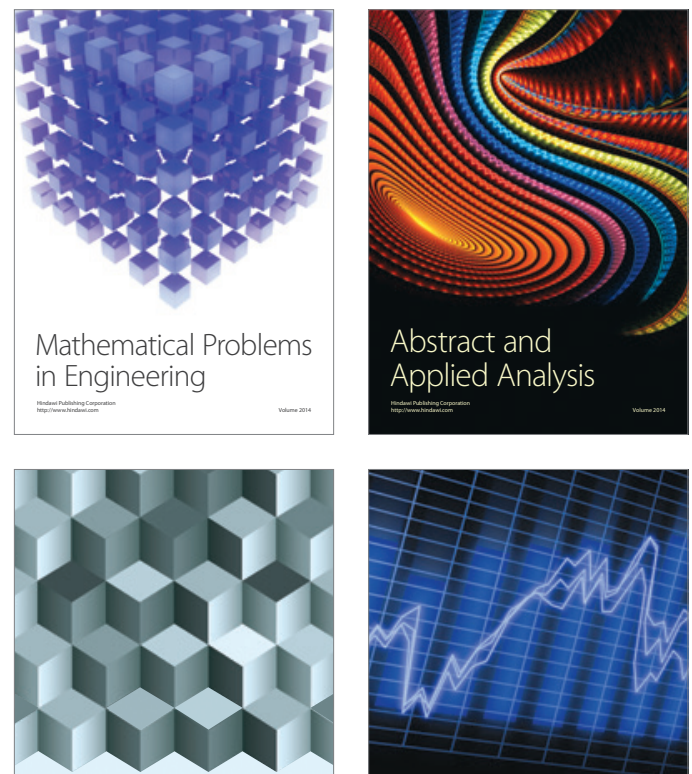

Journal of

Function Spaces

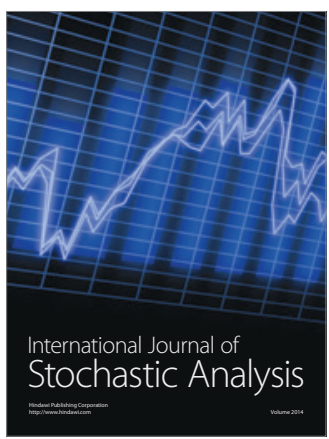

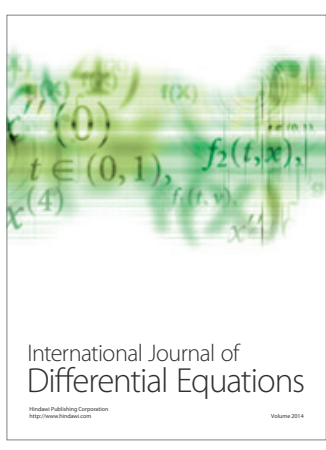
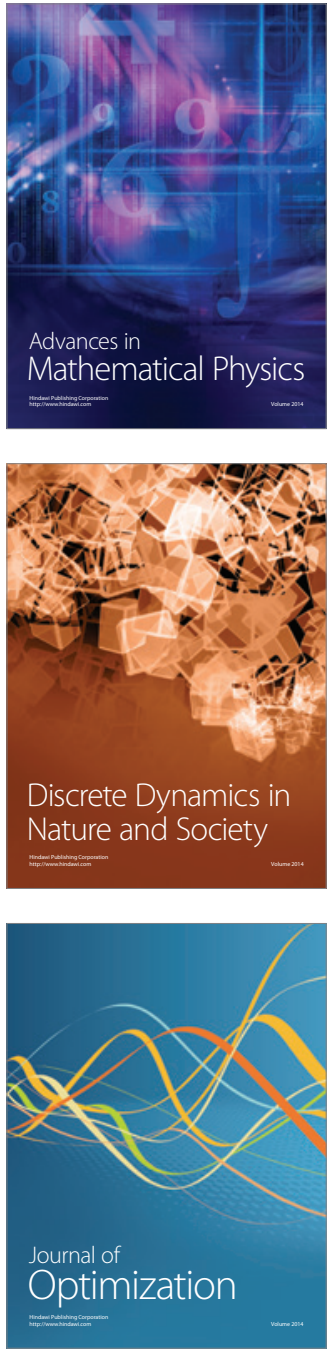